\title{
Magnitude and factors associated to physical violence among house maids of Mekelle town, Tigray, northern, Ethiopia: A cross sectional study
}

\author{
Milete Zenebe ${ }^{1}$, Azeb Gebresilassie ${ }^{2}$, Huruy Assefa ${ }^{2, *}$ \\ ${ }^{1}$ Student at Department of Public Health, College of Health Sciences, Mekelle, Tigray, Ethiopia \\ ${ }^{2}$ Department of Public Health, College of Health Sciences, Mekelle University, PO. Box, 1871 Mekelle, Ethiopia
}

Email address:

huruyame@yahoo.com (H. Assefa)

\section{To cite this article:}

Milete Zenebe, Azeb Gebresilassie, Huruy Assefa. Magnitude and Factors Associated to Physical Violence among House Maids of Mekelle Town, Tigray, Northern, Ethiopia: A Cross Sectional Study. American Journal of Nursing Science. Vol. 3, No. 6, 2014, pp. 105-109.

doi: 10.11648/j.ajns.20140306.13

\begin{abstract}
Background: Housemaids are part of women's with low socioeconomic status and most of their backgrounds are either with poor or divorced family and/or dead parents. They also spent their whole time on domestic work. Housemaid woman can experience physical violence. Therefore, the aim of the study is assess the magnitude and factors related to physical violence among house maids. Objective: To assess the magnitude and factors related to physical violence among house maids of Mekelle town, Tigray, Ethiopia. Methodology: Community based cross sectional study design with multistage sampling were conducted among female housemaids in Mekelle town Tigray, Ethiopia. The data was analyzed using SPSS. Using logistic regression crude and adjusted odds ratio with $95 \%$ confidence interval were calculated. Result: A total of 595 housemaid's had participated with a response rate of $94 \%$. In this finding the prevalence of physical violence was $16.3 \%$. This finding showed that respondents' family live together [AOR $=0.41 ; 95 \%$ CI: $0.20-0.85$ ], employers drink alcohol [AOR=7.50; 95\% CI: 4.21-13.32] and when employers drink any alcohol [AOR=3.47; CI: 1.04-11.53] were significant predictors of physical violence among housemaids. Conclusion: The prevalence of physical violence was high. Drinking alcohol and housemaids living with their family were the determinant factors for physical violence among housemaids.
\end{abstract}

Keywords: Physical Violence, Housemaid, Community Based

\section{Introduction}

WHO defines Violence against Women "any act of GBV that results in, or is likely to result in, physical, sexual or psychological harm or suffering to women, including threats of such acts, coercion or arbitrary deprivation of liberty, whether occurring in public or private life [1,2].

The seriousness in magnitude of physical violence globally, and lack of information on the dimensions and context of the problem in Ethiopia is very visible [3]. Women have the right to maternity leave with full pay. But this opportunity is difficult to find for housemaid women due to their economical ties with their employer and social stigmatization [4].

According to studies found out, the overall magnitude of physical violence against married women is about $45 \%$. Of these, $53 \%$ reported minor and serious somatic injuries in their lifetime. [3, 4]. Ethiopia has one of the highest prevalence of women's with physical violence [5]. Woman's physical violence is associated with an increased risk for sexual and physical problems and its consequences [6].

Physical violence occurs when someone uses a part of their body or an object to control a person's actions. Sexual violence occurs when a person is forced to unwillingly take part in sexual activity.

It is known that housemaid with a physical violence are at risk for unwanted pregnancy, unsafe abortion and other health problems like sexually transmitted infections including HIV. This happens mostly to women's in developing countries like Ethiopia in which women's with low socio economic status.

Most backgrounds of these housemaids are from either poor or divorced family or dead parents in which they may not get the opportunity to learn or other additional source of income. Most of the time employers do not give focus and may not consider employees personal life. Those housemaids 
almost spent their whole time to domestic work. Their working hour is long but with very low monthly payments, as a result economical improvement through this payment is impossible. Consequently, future life of housemaids may need to be considered. They are also part of physical violence either by their employers or any one from employer family.

Although there are many researches done on gender based violence in the world, including our country Ethiopia, there is a serious lack of adequate and reliable information on the magnitude, as well as factors associated with physical violence among housemaids. This study is expected to fill the gap by assessing the magnitude and factors associated with physical violence among house maids living in Mekelle town, Tigray, Ethiopia.

\section{Methods and Materials}

Community based cross sectional study design was conducted among the housemaids of Mekelle town from January 27-march7, 2014. Mekelle is the regional city of the Tigray and far $783 \mathrm{~km}$ north of Addis Ababa, the capital city of Ethiopia. According to the census of 2010 the estimated population size of the town reaches 215,910 .

A total of 634 respondents using multistage sampling technique were calculated using single population proportion formula by assuming prevalence of $49 \%$ [7], 95\% confidence interval, 10\% non-response rate and 1.5 design effect.

Data on Socio-demographic characteristics, Salary, Work experience, House maids agreement of employment with employer being contract or permanent, Employers background information(educational, occupation, family size, religion) and Family history of the employee (family near-by, family help, marital status and educational status of family, estimated income level) were collected using a pretested questionnaire.

The questionnaire was translated in to local language (Tigrigna) and back to English by professionals. Data collection was conducted by trained six female nurse data collectors and the supervisors. Training was given to data collectors and supervisors about the objective of the study and confidentiality. The investigators and the supervisors was strictly followed the overall activities and completeness of the questionnaire. Pre-test was done on $5 \%$ of the sample size at other place which is similar with the study area.

Physical violence was measured through six items. These are intentional acts 'yes' to pushing and slapping; beating, kicking/dragging, burning/chocking, and threatening by the respondents. Then physical violence was computed by aggregating these six items using SPSS version 16 statistical soft ware. At least one 'Yes 'response among the six items qualifies the respondent for being faced with any form of physical violence.

\section{Data Analysis}

Data were entered into Epi-Info version 3.5.1 and exported to SPSS version 20.0 statistical package for analysis. The results were presented in the form of tables and text using frequencies and summary statistics such as mean and standard deviation for continuous variables and percentages for categorical variables to describe the study population in relation to relevant variables. The data were analyzed using logistic regression to determine the determinant factors on the outcome variable and to control confounding. All variables having a $p$-value $\leq 0.05$ in the bivariate analysis were further entered into multivariable logistic regression model. Variables having p-value $\leq 0.05$ in the multivariable logistic regression were statistically significant. Crude and adjusted odds ratios with their $95 \%$ confidence intervals were calculated.

\section{Operational Definitions}

- $\quad$ Physical Violence: is any form of violent act which can result in physical harm including slapping, punching, kicking/drugging, beating/hitting with any object, burning/chocking, and threatening using a knife or a gun etc) against female house maids.

- Housemaids: Females who are working either contract (daily worker) or permanent at house holds

\section{Ethical Consideration}

Ethical clearance was obtained from Ethical Review Committee of Mekelle University, College of Health Sciences and department of public health. An official permission letter was obtained from Mekelle Health Office. Informed verbal consent was obtained from study participants in their local language after explaining the purpose of the study, potential risks and benefits of partaking in the study, and the right to withdraw from the study at any time. The participants were also assured about the confidentiality of the data.

\section{Result}

\subsection{Socio-Demographic Characteristics of House Maids}

From the total study participants, 595 households had participated in the study with a response rate of $94 \%$. About half of the study participants were in the age group of 15-19 years. Majority were Orthodox $(97.5 \%)$ in religion and single $(85.5 \%)$. Out of the total study participants $43.5 \%$ were illiterate and $72.2 \%$ of the respondents had $100-300$ monthly income. [Table 1]

Table 1. Socio-demographic characteristics of housemaids in Mekelle town 2014 G.C Tigray Ethiopia ( $n=595)$.

\begin{tabular}{lll}
\hline Variables & Frequency & Percent \\
\hline Age in years & & \\
$<15$ & 109 & 18.3 \\
$15-19$ & 303 & 50.9 \\
$20-24$ & 101 & 17 \\
$25-29$ & 62 & 10.4 \\
$30-34$ & 14 & 0.4 \\
\hline
\end{tabular}




\begin{tabular}{lll}
\hline Variables & Frequency & Percent \\
\hline $35-39$ & 5 & 0.8 \\
$40+$ & 7 & 0.2 \\
Marital status & & \\
Divorced & 35 & 5.9 \\
Live with friend & 4 & 0.7 \\
Married & 42 & 7.1 \\
Single & 509 & 85.5 \\
Widowed & 5 & 0.8 \\
Religion & & \\
Catholic & 11 & 1.3 \\
Orthodox & 580 & 97.5 \\
Protestant & 2 & 0.3 \\
Educational status of respondents & & \\
$1-6$ & 135 & 22.7 \\
$7-8$ & 104 & 17.5 \\
$9-12$ & 79 & 13.3 \\
Diploma & 5 & 0.8 \\
Degree and above & 1 & 0.2 \\
Illiterate & 257 & 43.2 \\
Read and write & 12 & 2 \\
Monthly income & & \\
100-300 & 430 & 72.2 \\
301-450 & 130 & 21.8 \\
$>450$ & 35 & 5.9 \\
\hline
\end{tabular}

Table 2. Housemaid characteristics in Mekelle town Tigray Ethiopia $(n=595)$.

\begin{tabular}{lll}
\hline Variable & Frequency & Percent \\
\hline Family live situation & & \\
Divorced & 94 & 15.8 \\
Both dead & 4 & 6.9 \\
Only father alive & 34 & 5.7 \\
Only mother alive & 52 & 8.7 \\
live together & 374 & 62 \\
Nearby family & & \\
No & 267 & 44.9 \\
Yes & 328 & 55.1 \\
Mother education & & \\
1-6 & 35 & 5.9 \\
7-8 & 17 & 2.9 \\
9-12 & 14 & 2.4 \\
Degree and above & 1 & 0.2 \\
Diploma & 5 & 0.8 \\
Illiterate & 437 & 73.4 \\
Read and write & 85 & 14.3 \\
Family income & & \\
High & 13 & 2.2 \\
Low & 367 & 61.7 \\
Medium & 215 & 36.1 \\
You believe you have enough salary & & \\
No & 394 & 66.2 \\
Yes & 201 & 33.8 \\
Get family support & & \\
No & 335 & 56.3 \\
Yes & 260 & 43.7 \\
Father education & & \\
1-6 & 30 & 5 \\
7-8 & 11 & 1.8 \\
9-12 & 7 & 1.2 \\
Diploma & 2 & 0.3 \\
Read and write & 504 & 84.7 \\
\hline & 4 & 6 \\
\hline
\end{tabular}

\subsection{Household Characteristics of the Respondents}

Study participants were asked about current status of their family life. More than six from ten of the respondents' family was living together and had low family income. More than half of the respondents were living with their family and did not get support from their family. Three fourth of the respondents fathers were illiterate and majority of the respondents mothers $(84.7 \%)$ were illiterate. [Table 2]

\subsection{Characteristics of Employers}

When we look on the characteristics of employers of the housemaid 277(46.6\%) were diploma and above and small number of the employers were illiterate, secondary education, read and write. More than six from ten of their employers were medium in economic status. Small number $(23.9 \%)$ of the employers was drink alcohol. [Table 3]

Table 3. Characteristics of employee of the servants in Mekelle town Tigray Ethiopia 2014 ( $n=595)$.

\begin{tabular}{lll}
\hline Variables & Frequency & Percentage \\
\hline When chew chat & & \\
1 or 2 weekly & 9 & 1.5 \\
Once a day & 4 & 0.7 \\
Sometimes & 14 & 2.4 \\
Employee cigarette smoke & & \\
No & 570 & 95.8 \\
Yes & 25 & 4.2 \\
How many times smoke & & \\
1-2 weekly & 3 & .5 \\
Once daily & 17 & 2.9 \\
Sometimes & 4 & 0.7 \\
When they drink & & \\
$1-2$ weekly & 16 & 2.7 \\
1-3 monthly & 3 & 0.5 \\
Once daily & 21 & 3.5 \\
Sometimes & 102 & 17.1 \\
\hline
\end{tabular}

\subsection{Magnitude of Physical Violence}

The study revealed that the magnitude of physical violence among housemaid was $16 \%$. Majority of the respondents were pushed by force, hit by box, pulled by employers and forced to be angry. [Table 4]

Table 4. Physical violence in Mekelle town, Tigray, Ethiopia 2014 ( $n=595)$.

\begin{tabular}{lll}
\hline Variables & Frequency & Percent \\
\hline Any punishment from employer & 512 & 86.1 \\
No & 83 & 14 \\
Yes & & \\
Pushed by force & 519 & 87.2 \\
No & 76 & 12.7 \\
Yes & & \\
Hit by box & 527 & 88.6 \\
No & 68 & 11.4 \\
Yes & & \\
Pulled by employer & 541 & 90.9 \\
No & 54 & 9.1 \\
Yes & & \\
Forced to be angry & 547 & 91 \\
No & 48 & 8.1 \\
Yes & & 83.7 \\
Physical violence & 498 & 16.3 \\
No & 97 & \\
Yes &
\end{tabular}




\subsection{Factors Associated with Physical Violence among Housemaids}

On the multivariate logistic regression analysis, respondents' family live together, employers drink alcohol and when employers drink any alcohol were significant predictors of physical violence among housemaids.

This finding showed that housemaids live together were 59\% less likely getting physical violence than housemaids having divorced family [AOR $=0.41 ; 95 \% \mathrm{CI}: 0.20-0.85]$.

The other predictor variable for physical violence was employers drink alcohol. Employers drinking any alcohol were 7.49 times more likely to violence with the housemaids than those not drinking any alcohol $[\mathrm{AOR}=7.50 ; 95 \% \mathrm{CI}$ : 4.21-13.32].

In addition, those employers drink alcohol every day were 3.47 times more violent to their housemaids than employers drink alcohol 1-2 weekly [AOR=3.47; CI:1.04-11.53].

Table 5. Bivariate and Multivariate analysis between dependent and independent variable among house servants in Mekelle town 2014 ( $n=595$ ).

\begin{tabular}{|c|c|c|c|c|}
\hline \multirow{2}{*}{ Variable } & \multicolumn{2}{|c|}{ Physical violence } & \multirow{2}{*}{$\begin{array}{l}\text { Crude } \\
\text { OR[95\%CI] }\end{array}$} & \multirow{2}{*}{$\begin{array}{l}\text { Adjusted } \\
\text { OR[95\%CI] }\end{array}$} \\
\hline & Yes $(\%)$ & No $(\%)$ & & \\
\hline \multicolumn{5}{|l|}{ Attending school now } \\
\hline No & $15(12.3)$ & $107(87.7)$ & 1 & 1 \\
\hline Day time & $76(16.5)$ & $384(83.5)$ & $4.3(1.42-13.23)$ & $4.15(0.97-17.8)$ \\
\hline Night time & $7(53.8)$ & $6(46.2)$ & $0.71(0.40-1.30)$ & $0.96(0.49-1.90)$ \\
\hline \multicolumn{5}{|l|}{ Husband education } \\
\hline $1-8$ grade & $5(20.8)$ & $19(79.2)$ & 1 & 1 \\
\hline 12 grade and above & $9(75)$ & $3(25)$ & $1.41(0.51-3.87)$ & $1.81(0.56-5.88)$ \\
\hline $9-12$ grade & $4(50)$ & $4(50)$ & $1.79(0.47-6.73)$ & $0.65(0.12-3.59)$ \\
\hline I do not know & $2(66.7)$ & $1(33.3)$ & $5.4(1.3-21.84)$ & $3.47(0.55-22.10)$ \\
\hline Illiterate & $19(90.5)$ & $2(9.5)$ & $2.7(0.24-29.9)$ & $1.65(0.09-30.85)$ \\
\hline Read and write & $6(100)$ & $0(0)$ & $0.56(0.13-2.47)$ & $0.39(0.07-2.31)$ \\
\hline \multicolumn{5}{|l|}{ Family live together } \\
\hline Divorced & $22(23.4)$ & $72(76.6)$ & 1 & 1 \\
\hline None alive & $7(17.1)$ & $34(82.9)$ & $0.67(0.26-1.73)$ & $0.52(0.16-1.70)$ \\
\hline Only father alive & $7(20.6)$ & $27(79.4)$ & $0.85(0.33-2.21)$ & $0.78(0.24-2.55)$ \\
\hline Only mother alive & $11(21.2)$ & $41(78.8)$ & $0.88(0.39-1.99)$ & $0.74(0.27-1.98)$ \\
\hline Yes & $50(13.4)$ & $324(86.6)$ & $0.51(0.29-0.89)$ & $0.41(0.19-0.85) *$ \\
\hline \multicolumn{5}{|l|}{ Get family support } \\
\hline No & $44(13.1)$ & 291(86.9) & 1 & 1 \\
\hline Yes & $53(20.4)$ & $207(79.6)$ & $1.69(1.09-2.62)$ & $1.57(0.92-2.69)$ \\
\hline \multicolumn{5}{|c|}{ Any drink of employer } \\
\hline No & $34(7.5)$ & $419(92.5)$ & 1 & 1 \\
\hline Yes & $63(44.4)$ & $79(55.6)$ & $9.83(6.07-15.90)$ & $7.50(4.21-13.32) *$ \\
\hline \multicolumn{5}{|l|}{ When chat chew } \\
\hline 1 or 2 weekly & $2(22.2)$ & $7(77.8)$ & 1 & 1 \\
\hline Once a day & $3(75.0)$ & $1(25)$ & $17.29(1.78-1.2)$ & $0.278(0.02-4.09)$ \\
\hline Sometimes & $8(57.1)$ & $6(42.9)$ & $7.68(2.60-22.7)$ & $2.714(0.15-50.15)$ \\
\hline \multicolumn{5}{|c|}{ How many times smoke } \\
\hline 1-2 weekly & $2(66.7)$ & $1(33.3)$ & 1 & 1 \\
\hline Once daily & $11(64.7)$ & $6(35.3)$ & $11.76(1.05-131.15)$ & $2.23(0.11-47.51)$ \\
\hline Sometimes & $1(25)$ & $3(75)$ & $10.78(3.881-29.94)$ & $3.01(0.61-14.82)$ \\
\hline \multicolumn{5}{|c|}{ When employers drink } \\
\hline 1-2 weekly & $8(50)$ & $8(50)$ & 1 & 1 \\
\hline 1-3 times monthly & $1(33.3)$ & $2(66.7)$ & $6.2(0.55-69.70)$ & $1.21(0.35-4.18)$ \\
\hline 1-2 weekly & $15(71.4)$ & $6(28.6)$ & $30.81(11.23-84.52)$ & $1.31(0.085-20.352)$ \\
\hline Every day & $39(38.2)$ & $63(61.8)$ & $7.63(4.487-12.97)$ & $3.47(1.04-11.53)^{*}$ \\
\hline
\end{tabular}

\section{Discussion}

The study has tried to assess the prevalence and determinant factors of physical violence among house maids residing in Mekelle town. This study has showed that the prevalence of physical violence among house maids was $16 \%$. This prevalence was higher than the study done in Ethiopia $(5 \%)$ [8]. This might be due to the fact that Mekelle is one of the towns which have many housemaids which encounter physical violence. Another reason might be due to the fact that the national survey includes rural areas which have small number of housemaids. Another possible reason might be women sometimes learn to "accept" violence in situations where they are victims.

This study revealed that large proportion (90\%) of house maids lived with illiterate husbands had physical violence at least once in their life time. This showed that as most studies indicated illiterate people has low capacity to read and understood things easily [9]. This might restrict them from knowing their rights and responsibilities as a result they might accept physical violence as gift from GOD and a chance of matter.

The study also showed that the proportion of house maid who get family support and had no physical violence was $79.6 \%$. This is true that the financial support from their family relieves tension and made them to resist and protect 
themselves from physical violence. The study showed that $55.6 \%$ and $77.8 \%$ of the employer were taking some kind of alcohol, chew chat and undertaken physical violence on their house maids, respectively. This was due to the fact that alcohol has depressive mental impairment and chat has mental excitement effect which encourages human beings to undertake physical violence against their house maids.

World Health Organization study of 24,000 women from 10 different countries with different cultures found that $15 \%$ $71 \%$ had experienced physical or sexual violence from their partners at some point in their lifetime. This prevalence also lower than that of WHO findings, this might be due to the fact that Ethiopia has low number of housemaids as a result of its low economic status.

No single factor alone can 'cause' violence against women, rather a number of interrelated factors work in combination at different levels increasing the likelihood of individual to engage in a violent action [10]. This study revealed that the most common socio-cultural risk factor for physical violence, as reported by the respondents, was alcohol drink. House servants in which their employee drunk alcohol everyday were more likely to get physical violence and house servants whose employee drunk alcohol were more likely to get physical violence. This finding was similar with study conducted in USA showed that Antisocial behaviours, attitudes , beliefs, Use of drugs, tobacco or alcohol, Aggressive behaviour in early development, Attention deficits, hyperactivity or learning disorders, Behaviour control problems and Social problems were determinant factors of physical violence [11]. This might be due to the fact that alcohol has central nervous system impairment property which leads the employee to undertake physical violence on their housemaids.

The study was also revealed that housemaids live with family significantly associated with physical violence of the housemaids. The possible explanation for this might be due to the fact if family are living together the house maids have psychological confidence enough on their right protection against the physical violence from their employee. Another reason might be the employee could have fear of law and her family. Studies done before showed that maternal education, paternal education and economic status of the family were determinant factors for physical violence but this study does not show an association.

In addition to the significant contribution of our findings, the study has limitations, which have social desirability and recall bias as natures of cross-sectional study design. There may be some biases to give accurate information since they afraid of their employers.

\section{Conclusion and Recommendation}

The prevalence of physical violence among the housemaids was high. The determinant factors of physical violence were drinking alcohol and housemaids living with their family.

Empowering housemaid through education, and improving their ability to find employment and income, along with increasing public awareness of human rights issues through education could lower the prevalence of physical violence. Also encouraging men to allow their housemaids to be more actively involved in decision making is another important step that could help reduce physical violence against housemaids. Increasing public awareness of human rights through education and outreach programs is a very important measure that should be undertaken through reliable local resources within communities.

\section{References}

[1] Zubairu I, Prevalence and correlates of gender-based violence among female university students in Northern Nigeria, African Journal of Reproductive Health, Vol. 15, No. 3, Sept, 2011, pp. 123-133.

[2] Yakin Ertürk, 15 years of The United Nations Special RaPporTeur on Violence against Women, Its Causes And Consequences Stockholm (12 September 2008).

[3] Deyessa N, Magnitude, type and outcomes of physical violence against married women in Butajira, southern Ethiopia, Apr 1998; 36(2):83-92.

[4] Country WID Profile (Ethiopia), Japan International Cooperation Agency Planning Department, December 1999.

[5] Yemane B. Ending Domestic Violence against Women in Ethiopia. EthiopJHealth Dev2004; 18(3):131-3.

[6] Thompson A. 15 years of The United Nations Special Rapporteur on Violence against Women, Its Causes and Consequences: UN, 2009).

[7] Sexual and Gender Based violence in Africa, Sub Saharan African Region, Population counsel, the population counsel Inc.2008 WWW.popcounsel.org.

[8] Rosa Bennathan, Acid Violence, In Conversation with Shabina Begum, October 24, 2013.

[9] Mohamed Motala, Domestic Workers in South Africa: its Modern Day Slavery, 3 May 2010.

[10] World Health Organization, WHO multi-country study on women's health and domestic violence against women: summary report of initial results on prevalence, health outcomes and women's responses. Geneva, World Health Organization, 2005. Available at: http://www.who.int/gender/violence/whomulticountrystudy/en /. Accessed December 26, 2014.

[11] C. Garcia-Moreno, H. A. Jansen, M. Ellsberg, L. Heise, and C. $\mathrm{H}$. Watts, "Prevalence of intimate partner violence: findings from the WHO multi-country study on women's health and domestic violence," The Lancet, vol. 368, no. 9543, pp. 1260 1269, 2006. View at Publisher . View at Google Scholar $\cdot$ View at Scopus 